\title{
Carotenoid intakes and risk of breast cancer defined by estrogen receptor and progesterone receptor status: a pooled analysis of 18 prospective cohort studies ${ }^{1-3}$
}

\author{
Xuehong Zhang, Donna Spiegelman, Laura Baglietto, Leslie Bernstein, Deborah A Boggs, Piet A van den Brandt, \\ Julie E Buring, Susan M Gapstur, Graham G Giles, Edward Giovannucci, Gary Goodman, Susan E Hankinson, \\ Kathy J Helzlsouer, Pamela L Horn-Ross, Manami Inoue, Seungyoun Jung, Polyna Khudyakov, Susanna C Larsson, \\ Marie Lof, Marjorie L McCullough, Anthony B Miller, Marian L Neuhouser, Julie R Palmer, Yikyung Park, Kim Robien, \\ Thomas E Rohan, Julie A Ross, Leo J Schouten, James M Shikany, Shoichiro Tsugane, Kala Visvanathan, \\ Elisabete Weiderpass, Alicja Wolk, Walter C Willett, Shumin M Zhang, Regina G Ziegler, and Stephanie A Smith-Warner
}

\begin{abstract}
Background: Epidemiologic studies examining associations between carotenoid intakes and risk of breast cancer by estrogen receptor (ER) and progesterone receptor (PR) status are limited.

Objective: We investigated these associations in a pooled analysis of 18 cohort studies.

Design: Of 1,028,438 participants followed for a maximum followup of $26 \mathrm{y}$ across studies, 33,380 incident invasive breast cancers were identified. Study-specific RRs and 95\% CIs were estimated by using Cox proportional hazards regression and then pooled by using a random-effects model.

Results: $\alpha$-Carotene, $\beta$-carotene, and lutein/zeaxanthin intakes were inversely associated with the risk of ER-negative (ER-) breast cancer (pooled multivariable RRs of the comparison between the highest and lowest quintiles): $\alpha$-carotene $(0.87 ; 95 \%$ CI: $0.78,0.97)$, $\beta$-carotene (0.84; 95\% CI: 0.77, 0.93), and lutein/zeaxanthin (0.87; 95\% CI: $0.79,0.95)$. These variables were not inversely associated with the risk of ER-positive $(\mathrm{ER}+)$ breast cancer (pooled multivariable RRs for the same comparison): $\alpha$-carotene (1.04; 95\% CI: 0.99, 1.09), $\beta$-carotene (1.04; 95\% CI: 0.98, 1.10), and lutein/zeaxanthin $(1.00 ; 95 \%$ CI: $0.93,1.07)$. Although the pooled RRs for quintile 5 for $\beta$-cryptoxanthin were not significant, inverse trends were observed for ER - and ER+ breast cancer $(P$-trend $\leq 0.05)$. Nonsignificant associations were observed for lycopene intake. The associations were largely not appreciably modified by several breast cancer risk factors. Nonsignificant associations were observed for PR-positive and PR-negative breast cancer.
\end{abstract}

Conclusions: Intakes of $\alpha$-carotene, $\beta$-carotene, and lutein/zeaxanthin were inversely associated with risk of ER-, but not ER+, breast cancer. However, the results need to be interpreted with caution because it is unclear whether the observed association is real or due to other constituents in the same food sources. Am J Clin Nutr 2012;95:713-25.

\section{INTRODUCTION}

Carotenoids are fat-soluble pigments present in red, yellow, orange, and dark-green fruit and vegetables. More than 600 carotenoids occur in nature, with the most commonly studied carotenoids being $\alpha$-carotene, $\beta$-carotene, $\beta$-cryptoxanthin, lutein, zeaxanthin, and lycopene because of their abundance in the diet and comparatively high concentrations in plasma (1). Car-

\footnotetext{
${ }^{1}$ From the Departments of Nutrition (XZ, EG, SJ, WCW, and SAS-W), Epidemiology (XZ, DS, JEB, EG, SEH, WCW, and SAS-W), and Biostatistics (DS and PK), Harvard School of Public Health, Boston, MA; The Cancer Epidemiology Centre, Cancer Council Victoria, Carlton, Victoria, Australia (L Baglietto); the Centre for Molecular, Environmental, Genetic and Analytic Epidemiology, University of Melbourne, Victoria, Australia (L Baglietto and GGG); the Division of Cancer Etiology, Department of Population Science, Beckman Research Institute and City of Hope National Medical Center, Duarte, CA (L Bernstein); the Slone Epidemiology Center at Boston University, Boston, MA (DAB and JRP); the Department of Epidemiology, GROW-School for Oncology and Developmental Biology, Maastricht University, Maastricht, Netherlands (PAvdB and LJS); the Division of Preventive Medicine, Department of Medicine, Brigham and Women's Hospital and Harvard Medical School, Boston, MA (JEB and SMZ); the Epidemiology Research Program, American Cancer Society, Atlanta, GA (SMG and MLM); the Channing Laboratory, Department of Medicine, Brigham and Women's Hospital and Harvard Medical School, Boston, MA (EG, SHE, and WCW); the Division of Public Health Sciences, Fred Hutchinson Cancer Research Center, Seattle, WA (GG and MLN); The Prevention and Research Center, The Weinberg Center for Women's Health and Medicine, Mercy Medical Center, Baltimore, MD (KJH); the Department of Epidemiology, The Johns Hopkins Bloomberg School of Public Health, Baltimore, MD (KJH and KV); the Cancer Prevention Institute of California, Fremont, CA (PLH-R); the Epidemiology and Prevention Division, Research Center for Cancer Prevention and Screening, National Cancer Center, Tokyo, Japan (MI and ST); the Division of Nutritional Epidemiology, National Institute of Environmental Medicine, Karolinska Institute, Stockholm, Sweden (SCL and AW); the Department of Medical Epidemiology and Biostatistics, Karolinska Institute, Stockholm, Sweden (ML); The Dalla Lana School of Public Health, University of Toronto, Toronto, Canada (ABM and TER); the Division of Cancer Epidemiology and Genetics, National Cancer Institute, Bethesda, MD (YP and RGZ); the Masonic Cancer Center, University of Minnesota, Minneapolis, MN (KR and JAR); The Division of Preventive Medicine, University of Alabama School of Medicine, Birmingham, AL (JMS); the Institute of Community Medicine, University of Troms $\varnothing$, Troms $\varnothing$, Norway (EW); the Department of Etiological Research, Cancer Registry of Norway, Oslo, Norway (EW); and the Department of Genetic Epidemiology, Folkhälsan Research Center, Helsinki, Finland (EW).
} 
otenoids have been hypothesized to protect against carcinogenesis (2) by inhibiting the ability of reactive oxygen species to induce DNA damage-a crucial step in carcinogenesis and neoplastic transformation (3-5). In addition, provitamin A carotenoids (including $\alpha$-carotene, $\beta$-carotene, and $\beta$-cryptoxanthin) can be metabolized to retinol, which is important for the control of cellular differentiation and proliferation and immunologic functions (6). Other specific mechanisms have been identified for some carotenoids. For example, lutein/zeaxanthin might reduce cell proliferation and $\beta$-carotene (7), and lycopene might inhibit estrogen receptor-mediated signaling of $17 \beta$-estradiol and attenuate its deleterious effect on breast cancer (7).

Epidemiologic evidence from $\sim 50$ case-control studies and 15 cohort studies examining the associations between carotenoid intakes and overall breast cancer risk has been inconsistent (8). The mixed results may be due in part to the fact that most epidemiologic studies have viewed breast cancer as a single disease. Indeed, breast cancer defined by hormone receptor status appears to be etiologically and clinically heterogeneous (9-13). $\mathrm{ER}^{4}$ and PR status are commonly used to define breast cancer subtypes, and the majority of early studies focused on only ER status (10). Because mechanisms independent of steroid hormones might play a more important role in ER - breast cancer (9-13), we hypothesized that carotenoid intakes might reduce risk of ER - breast cancer but not ER+ breast cancer, which is mainly influenced by hormones. We tested this hypothesis by examining the associations between intakes of specific carotenoids and risk of ER - breast cancer in the Pooling Project of Prospective Studies of Diet and Cancer (14) —an international consortium including $>1$ million women. We conducted secondary analyses by PR status or jointly by ER and PR status because these associations have received little attention to date (15-17).

\section{SUBJECTS AND METHODS}

\section{Study population}

For these analyses, we included 18 prospective cohort studies (18-35) that met the following inclusion criteria: ascertainment of $\geq 25$ incident cases of invasive ER - breast cancer and $\geq 25$ cases of invasive PR - breast cancer; publication of at least one diet and cancer analysis; assessment of long-term dietary intake, including intake of the 5 major dietary carotenoids; and evaluation of the validity of the dietary assessment method or a closely related instrument. Each included study was approved by the institutional review boards of the participating institutions.

\footnotetext{
${ }^{2}$ Supported by NIH grant CA055075 and a Breast Cancer Research Foundation grant.

${ }^{3}$ Address correspondence to X Zhang, Channing Laboratory at Landmark Center (West Wing), 401 Park Drive, Boston, MA 02115. E-mail: pooling @hsphsun2.harvard.edu.

${ }^{4}$ Abbreviations used: ER, estrogen receptor; ER+, estrogen receptor positive; ER-, estrogen receptor negative; FFQ, food-frequency questionnaire; $\mathrm{PR}$, progesterone receptor; $\mathrm{PR}+$, progesterone receptor positive; $\mathrm{PR}-$, progesterone receptor negative.

Received February 22, 2011. Accepted for publication December 9, 2011. First published online January 25, 2012; doi: 10.3945/ajcn.111.014415
}

\section{Ascertainment of breast cancer cases}

Incident invasive breast cancer cases were identified in each study through follow-up questionnaires and confirmed with subsequent medical record review $(22,31,33)$, linkage with cancer registries $(20,21,23,24,27,28,32,34-36)$, or both (19, 25, 26, 29, 30). Mortality registries were also used in some studies to ascertain additional cases $(19,20,22,25,26,29,33,35)$. Follow-up rates exceeded $90 \%$ for 16 of 18 current studies (14, $37-42$ ) included in this analysis and is $\geq 80 \%$ for all studies ( $37-$ 44). We used the receptor status data from each study to define breast cancer subtypes by ER/PR status. We classified the cases with borderline ER/PR status as being positive for that receptor. The overall proportion of missing of ER/PR status was $\sim 27 \%$ in this study.

\section{Dietary and nondietary assessment}

Each study assessed usual dietary intake by using a selfadministered FFQ and calculated daily consumption of each of the major carotenoids ( $\alpha$-carotene, $\beta$-carotene, $\beta$-cryptoxanthin, lutein/zeaxanthin, and lycopene; food sources only). Lutein and zeaxanthin were analyzed together because most food-composition databases report only a combined value for them $(1,45$, 46) because of laboratory difficulties in separating them. We calculated daily energy-adjusted carotenoid intakes by using the residual method (47). Total carotenoid intake was calculated by summing the intakes of the 5 specific carotenoids in each study. We also calculated a total carotenoid score. For the score, intake of each of the 5 carotenoids was categorized into quintiles ( 1 = lowest quintile, $5=$ highest quintile) and then the quintile scores for the 5 carotenoids were summed for each participant. The total carotenoid score ranged from 5 to 25 across participants.

Although the food intake estimates from the FFQ used in each study or a closely related instrument were compared with intakes estimated from multiple 24-h recalls or days of diet records, only a few of the calibration studies assessed intakes of specific carotenoids $(34,48-51)$. The correlation coefficients between $\beta$-carotene or carotene intakes estimated by the FFQ and comparison method ranged from 0.30 to 0.60 (34). The correlation coefficients between dietary intake estimated from the FFQ and plasma concentrations for the 5 major carotenoids ranged from 0.21 for lycopene to 0.48 for $\alpha$-carotene among nonsmokers in the Nurses' Health Study (48) and ranged from 0.28 for lycopene to 0.46 for $\beta$-cryptoxanthin in the Melbourne Collaborative Cohort Study (51).

$\beta$-Cryptoxanthin intake is positively correlated with dietary vitamin $\mathrm{C}$ intake (correlation coefficients ranged from 0.6 to 0.9 across studies) because both are concentrated in citrus fruit and fruit juices $(45,46)$. Correlations comparing vitamin $\mathrm{C}$ intake estimates from the FFQs with those from multiple 24-h recalls or diet records might serve as a reasonable surrogate of the validity of $\beta$-cryptoxanthin intake. The correlation coefficient between the FFQs used in these studies or closely related instruments and multiple days of dietary records or 24-h recalls ranged from 0.3 to 0.8 for vitamin C intake (18, $20,35,53,54)$. Information on nondietary factors was also collected by each study by using self-administered questionnaires at baseline. 


\section{Statistical analyses}

After applying the study-specific exclusion criteria, we further excluded participants with a history of cancer at baseline (except for nonmelanoma skin cancer) and who reported energy intakes $>3$ SDs from their study-specific $\log _{\mathrm{e}}$-transformed mean energy intake. We analyzed the Netherlands Cohort Study as a casecohort study, because dietary questionnaires were processed for only the cases and a random sample of the total cohort (55). We analyzed the Nurses' Health Study as 2 different cohorts [19801986, Nurses' Health Study (a); 1986-2006, Nurses' Health Study (b)] to take advantage of the more detailed dietary assessment available in 1986. These 2 blocks of person time, obtained from the same participants, are asymptotically uncorrelated according to the underlying theory of survival analysis (56), given that each person contributed only one outcome event and was then censored (ie, a woman who developed breast cancer in the 1980-1986 period would not be included in the 1986-2000 period).

We used Cox proportional hazards regression (57) to estimate study-specific RRs and 95\% CIs. We calculated person-years of follow-up from the date of questionnaire return to the date of diagnosis of incident invasive breast cancer, death, loss to followup (if applicable), or end of follow-up, whichever came first. We modeled age at baseline (in y) and year of questionnaire return as stratification variables to adjust simultaneously for age, calendar time, and time since entry into the study (14). In the multivariable analyses, we controlled for the following breast cancer risk factors: race-ethnicity, family history of breast cancer, personal history of benign breast disease, education, physical activity, BMI, height, age at menarche, age at first birth, parity, smoking habits, alcohol consumption, total energy intake, use of oral contraceptives, menopausal status at baseline, and postmenopausal hormone use among postmenopausal women. For each measured confounding variable in a study, we created a missing indicator variable for missing data because the proportion of missing data in the Pooling Project is generally low (14). We either adjusted for the abovementioned covariates directly in the model or we adjusted for confounders by using the propensity score method (58-60) when the number of cases of the outcome evaluated within a study was $<200$.

We pooled the study-specific RRs weighted by the inverse of their variances by using a random-effects model $(61,62)$ and tested for between-studies heterogeneity using the $Q$ statistic (62, 73). All statistical analyses were 2 -sided with a $P$ value of 0.05 indicating significance. We conducted all analyses by using the SAS software (version 9; SAS Institute Inc).

We conducted separate analyses for each carotenoid by using study-specific quintiles. The study-specific quintile cutoffs were based on the distributions in the subcohort in the case-cohort study and based on the baseline cohort distributions for the remaining studies. To calculate the $P$ value for the test for trend across categories, we used the median value for each intake category and modeled that variable as a continuous term. We tested for nonlinearity in the breast cancer association for each carotenoid by using restricted cubic splines $(64,65)$. In these analyses, we combined all studies into one data set, stratified by study, age, and year of questionnaire return and adjusted for the abovementioned confounding variables. We used a likelihood ratio test to compare the model including the linear and cubic spline terms selected by a stepwise regression procedure with the model including only the linear term for the carotenoid of interest. If the assumption of linearity held for the association between intake of a specific carotenoid and breast cancer risk, we further analyzed that carotenoid as a continuous variable.

We evaluated whether the observed association between intake of each carotenoid and breast cancer risk was modified by menopausal status at diagnosis (66) (premenopausal, postmenopausal), family history of breast cancer (yes, no), BMI (in $\mathrm{kg} / \mathrm{m}^{2} ;<25$, $\geq 25$ ), multivitamin use (yes, no), alcohol consumption (nondrinker, 1 to $<15 \mathrm{~g} / \mathrm{d}, \geq 15 \mathrm{~g} / \mathrm{d}$ ), smoking status (never, past, current), approximate median age at diagnosis ( $<64 \mathrm{y}, \geq 64 \mathrm{y}$ ), and follow-up period $(<5 \mathrm{y}, \geq 5 \mathrm{y})$ by using a mixed-effects meta-regression model (67). We used a contrast test (68) to examine whether the associations were differed significantly for subtypes of breast cancer defined by receptor status (ER - compared with $\mathrm{ER}+, \mathrm{PR}-$ compared with $\mathrm{PR}+$, and across the 4 subtypes jointly defined by ER/PR status).

We conducted further analyses to investigate the potential influence of measurement error on the associations between $\alpha$-carotene, $\beta$-carotene, $\beta$-cryptoxanthin, and lutein/zeaxanthin intakes and risk of ER - breast cancer. The method of Hamling et al (69) was used to obtain the expected cell counts of the $2 \times 2$ tables for the extreme quintile contrast after controlling for confounding, which produced RRs and 95\% CIs matching those of the original study-specific multivariate analyses. These $2 \times 2$ tables were then adjusted for estimates of measurement error in intake by the matrix method of Barron (70), and the $95 \%$ CIs were obtained to account for both the variance of the multivariate RRs obtained in the main study and the estimation of the misclassification probability in the study-specific validation study (71). Because few studies measured intake of the individual carotenoids in their validation studies, the misclassification matrix for total carotene intake was used as the best estimate of the expected misclassification in $\alpha$-carotene, $\beta$-carotene, and lutein/zeaxanthin intakes; dietary vitamin $\mathrm{C}$ intake was used to approximate the measurement error in $\beta$-cryptoxanthin intake. When carotene or dietary vitamin $\mathrm{C}$ intake was not assessed in the validation study for a study, we used the misclassification matrix from a similar FFQ; when this was not possible, the pooled misclassification matrix from the other validation studies was used. In some studies, the misclassification matrix produced one or more negative cell counts; when this occurred, we adjusted the misclassification matrix by the power transformation closest to but less than 1 (72). One validation study required an additional adjustment before the power transformation was taken (73).

\section{RESULTS}

During 7-26 y of follow-up of 1,028,438 participants in the 18 prospective cohort studies, 33,380 incident invasive breast cancer cases were identified with 7-26 y of follow-up in these 18 prospective cohort studies. More hormone receptor-positive breast cancer cases than hormone receptor-negative breast cancer cases were identified (19,282 ER+ compared with 4643 ERbreast cancers; 15,696 PR+ compared with 7203 PR - breast cancers; Table 1). Grouping the cases by ER and PR status, 14,849 were $\mathrm{ER}+\mathrm{PR}+, 3311$ were $\mathrm{ER}+\mathrm{PR}-, 640$ were $\mathrm{ER}-$ $\mathrm{PR}+$, and 3774 were $\mathrm{ER}-\mathrm{PR}-$. 


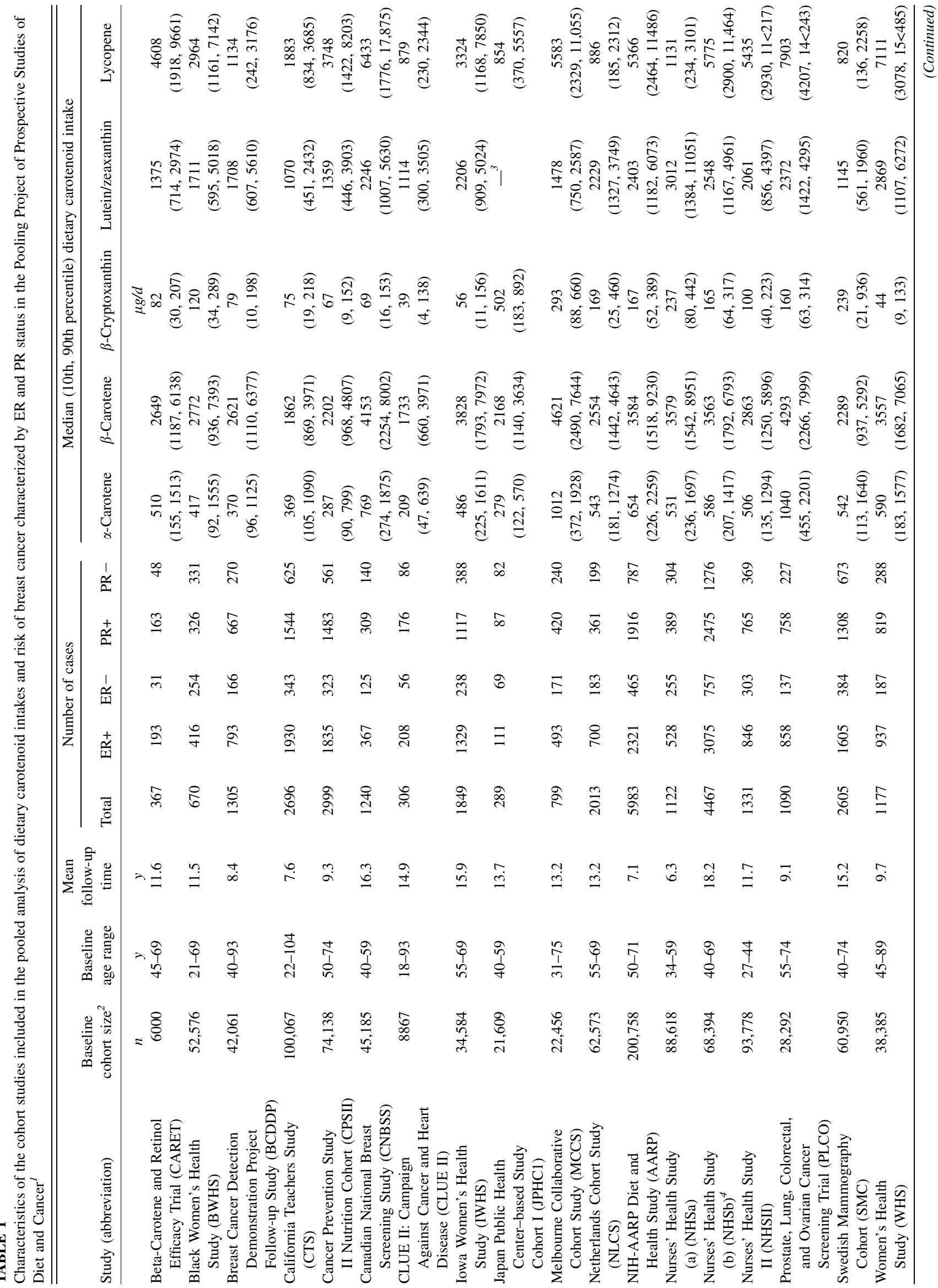


Large variability in carotenoid intake was observed across studies, with the range in the median intakes of the carotenoids varying from $\sim 3$-fold ( $\beta$-carotene) to 13 -fold ( $\beta$-cryptoxanthin) across studies (Table 1). The Pearson correlation coefficients comparing intakes of the 5 major carotenoids with one another ranged from $\sim 0.2-0.8$ across studies, with the highest correlations generally being observed between $\alpha$-carotene and $\beta$-carotene intakes $(r>0.5)$ and relatively lower correlations generally being observed for intake of other carotenoids $(r<0.3)$.

Because the age-adjusted results were similar to the multivariable-adjusted results, we present only the multivariable results. Of the 5 carotenoids evaluated, a significant association with risk of overall breast cancer was observed for only $\beta$-cryptoxanthin intake, and that association was relatively weak (pooled multivariable RR comparing the highest with lowest quintile $=0.95 ; 95 \%$ CI: $0.92,0.99 ; P$-trend $=0.01)$. For the remaining carotenoids, the pooled multivariable RRs comparing the highest with lowest quintile ranged from 0.98 to 1.00 (Table 2). For each carotenoid, the test for between-studies heterogeneity for the extreme quintile contrast was not significant (between-studies heterogeneity test, $P \geq 0.05$ for all; data not shown). We also conducted analyses in which we modeled intake of each carotenoid as a continuous variable, because no nonlinearity was evident in the associations between intakes of each carotenoid and risk of overall breast cancer (test for nonlinearity, $P>0.10$ ). The results were largely not significant (Table 2).

When we examined ER+ and ER - breast cancers separately, for $\alpha$-carotene, $\beta$-carotene, and lutein/zeaxanthin intakes, the risk of ER - breast cancer was 13-16\% lower for comparisons of the highest compared with the lowest quintiles, whereas null associations were observed for ER+ breast cancer (Table 2). Although the pooled RRs for quintile 5 for $\beta$-cryptoxanthin were borderline significant, inverse trends were observed for both ER - and ER+ breast cancer $(P$-trend $\leq 0.05)$. Total carotenoid intake was inversely associated with the risk of ER - breast cancer (pooled multivariable RR comparing the highest with lowest quintile: $0.86 ; 95 \%$ CI: $0.78,0.94$ ) but not with the risk of $\mathrm{ER}+$ breast cancer (pooled multivariable RR for the same comparison: 1.03 ; $95 \%$ CI: $0.99,1.08)$. Results were similar for the total carotenoid score derived by summing the quintile scores for each carotenoid (data not shown). Comparison of the highest with the lowest decile showed that the associations for ER - and ER+ breast cancer were similar in magnitude to those presented in Table 2 for the quintile analyses.

Because the tests for nonlinearity of the associations between intakes of the specific carotenoids and risk of ER - and ER+ breast cancers were not significant (test for nonlinearity, $P>$ 0.05 for all), we conducted additional analyses in which carotenoid intakes were modeled as continuous variables. The pooled multivariable RRs for the 5 major carotenoids ranged from 0.93 to 1.01 for an increment based on the approximate median difference between the study-specific 90th percentile and 10th percentile in intake (Table 2). As observed in the quintile analyses, there was a weak inverse association for $\beta$-carotene and lutein/zeaxanthin intakes with risk of ER - but not ER+ breast cancer (test for common effects by ER status, $P<0.05$ ). Nonsignificant associations were observed for $\alpha$-carotene, $\beta$-cryptoxanthin, and lycopene intakes for ER - and ER+ breast cancers. Further analysis restricted to whites yielded similar 


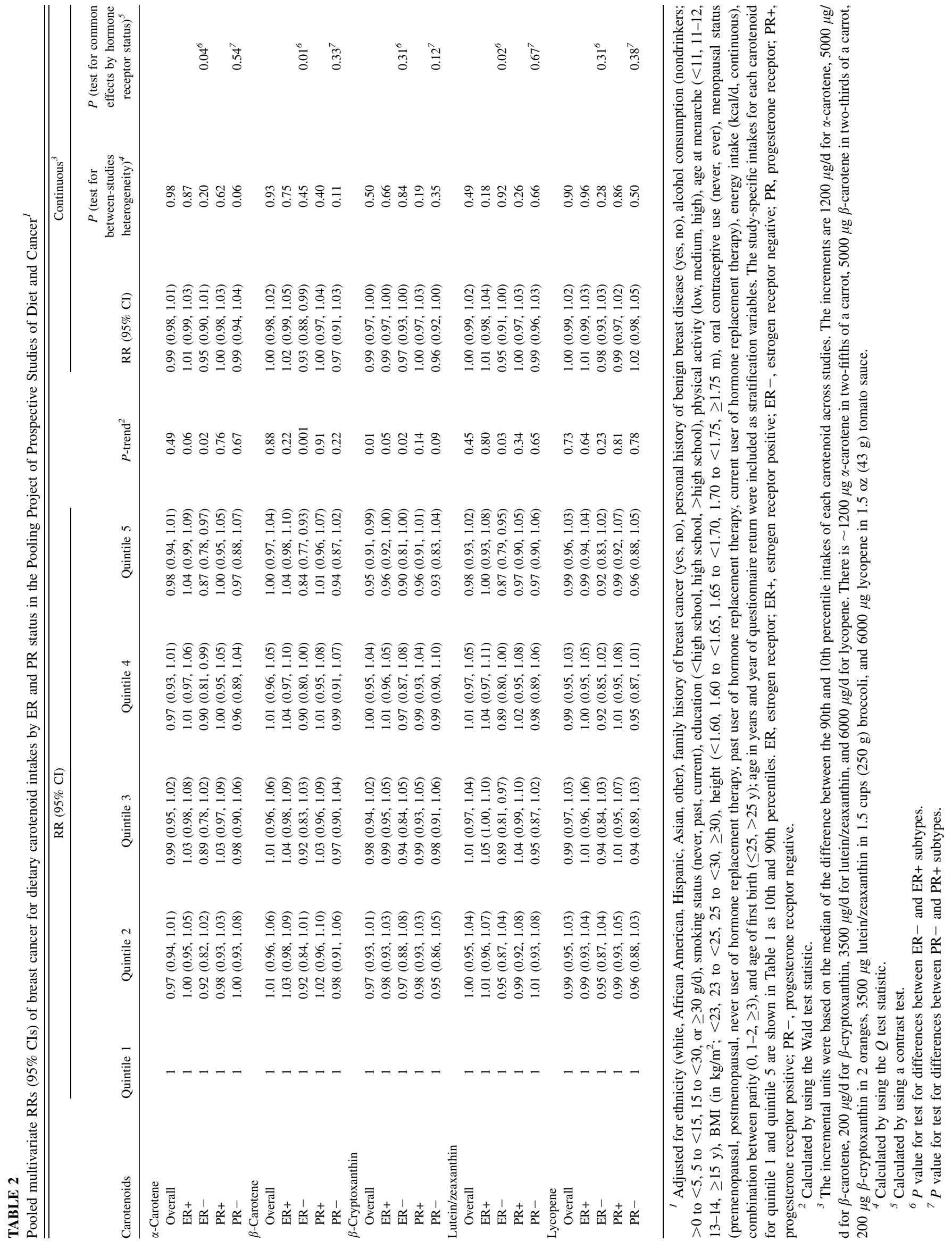


results (data not shown). Results were not significant for African Americans (304 ER - cases and $522 \mathrm{ER}+$ cases from 3 cohorts in our study). Despite the relatively smaller number of cases, associations of similar magnitude were observed for $\beta$-carotene and ER - breast cancer both in whites (pooled multivariable RR: 0.93; 95\% CI: 0.86, 1.00) and in African Americans (pooled multivariable RR: 0.89 ; 95\% CI: $0.71,1.11)$. The results for each carotenoid did not change materially after mutual adjustment of the 5 carotenoids (data not shown). In addition, results were essentially unchanged when we further adjusted for dietary folate and vitamin $\mathrm{C}$ intakes. Moreover, results on lycopene intake were similar when we restricted our analyses to studies in which consumption of tomato sauce or tomato products (for which the lycopene is more bioavailable) was assessed.

None of the carotenoids were significantly associated with the risk of $\mathrm{PR}+$ breast cancer in either the quintile or continuous analyses. Only $\beta$-cryptoxanthin (when modeled as a continuous variable) was significantly associated with risk of PR - breast cancer. There was no significant between studies heterogeneity for these results.

Intakes of each carotenoid were not associated with the risk of $\mathrm{ER}+\mathrm{PR}+$ or ER+ PR - breast cancer, with the pooled multivariable RRs comparing the highest with the lowest quintile ranging from 0.91 to 1.08 (Table 3). $\beta$-Carotene intake was modestly inversely associated with risk of ER - PR - breast cancer (pooled multivariable RR comparing the highest with the lowest quintile: 0.87 ; 95\% CI: 0.78, 0.96; $P$-trend $=0.01$ ). A similar inverse association was observed for lutein/zeaxanthin intake with the risk of ER - PR - breast cancer (pooled multivariable RR comparing the highest with the lowest quintile: $0.89 ; 95 \% \mathrm{CI}: 0.81,0.99 ; P$-trend $=0.16)$. In general, the strongest inverse associations were observed for risk of ER - PR+ breast cancer, although the CI for each carotenoid was wide because of the relatively small number of cases $(n=645)$ compared with the other subtypes.

We conducted further analyses to examine whether the associations with ER - and ER+ breast cancer differed by several breast cancer risk factors or factors that may interact with carotenoids by acting through the oxidative stress pathway. The associations between carotenoid intakes and risk of ER+ and ER - breast cancer were generally not significantly modified by menopausal status at diagnosis (Table 4), alcohol consumption (Table 5), smoking status (Table 5), family history of breast cancer (data not shown), BMI (data not shown), or multivitamin use (data not shown). In addition, results generally did not vary by age at diagnosis and follow-up time (data not shown). Because a relatively large number of comparisons were made in the stratified analyses, the results need to be interpreted with caution.

In analyses that corrected for misclassification in carotenoid intake estimates by using carotene or dietary vitamin $\mathrm{C}$ intake data in the validation studies to approximate the measurement error for the specific carotenoids, the risk estimates observed were substantially strengthened but less precise. The corrected pooled multivariable RRs (95\% CIs) comparing the highest with the lowest quintile of intake in relation to ER - breast cancer were $0.57(0.44,0.73)$ for $\alpha$-carotene, $0.70(0.54,0.90)$ for $\beta$-carotene, $0.81(0.67,0.99)$ for $\beta$-cryptoxanthin, and 0.75 $(0.53,1.07)$ for lutein/zeaxanthin. No significant between-studies heterogeneity was observed either before or after correction for misclassification in the intake estimates.

\section{DISCUSSION}

In general, no significant associations were observed between carotenoid intakes and risk of breast cancer overall and for ER+, $\mathrm{PR}+$, and $\mathrm{PR}-$ breast cancers. However, higher intakes of specific carotenoids were associated with a modest reduction in the risk of ER - breast cancer. These associations were largely not significantly modified by menopausal status, family history of breast cancer, alcohol consumption, smoking status, BMI, multivitamin use, median age at diagnosis, or follow-up time.

The relation between dietary carotenoid intakes and overall breast cancer risk has been investigated in $\geq 15$ cohort studies and 50 case-control studies with mixed results (8). A modest inverse association has been reported by most case-control studies, but most cohort studies have reported null results (8). Most case-control studies conducted before the mid-1990s reported inverse associations with $\beta$-carotene intake and breast cancer risk (8). However, these studies most likely measured multiple provitamin A carotenoids expressed in $\beta$-carotene equivalents rather than $\beta$-carotene itself, because comprehensive food-composition databases for individual carotenoids were not available until the mid-1990s $(46,48)$. Studies that evaluated intakes of the 5 major carotenoids reported null or weak inverse associations between intakes of each carotenoid and breast cancer risk, although the observed inverse associations have not been specific to a particular carotenoid (8). We generally observed null associations between intakes of the 5 major carotenoids and risk of overall breast cancer. No consistent results for a single carotenoid measured in plasma $(74,75)$ or breast adipose tissue (76) have been reported. Taken together, the associations between each of the major carotenoids (assessed either via questionnaire, in blood, or in adipose tissue) and overall breast cancer risk generally have been inconsistent. Treating breast cancer as a single disease might have contributed to the inconsistent results observed across studies because breast cancer subtypes defined by ER and PR status may be etiologically, as well as clinically, heterogeneous (9-13).

To date, relatively few studies examined associations between intakes of the 5 major carotenoids and risk of breast cancer according to ER/PR status, and most of these studies have been limited by a small number of cases for the less common subtypes (15-17). A relatively small population-based case-control study conducted in Australia in the early 1990s showed a stronger inverse association of $\beta$-carotene intake with risk of ER - breast cancer than with risk of ER+ breast cancer (15). Other studies reported only breast cancer subtypes defined by ER and PR status jointly. A population-based case-control study found no associations between intakes of the 5 major carotenoids and risk of any of the breast cancer subtypes defined jointly by ER/PR status (17). In contrast, the Women's Health Initiative Observational Study reported an $\sim 20 \%$ lower risk of $\mathrm{ER}+\mathrm{PR}+$ breast cancer for $\alpha$-carotene, $\beta$-carotene, and lycopene intakes comparing the highest with the lowest quintiles of intake, but null associations for intakes of these carotenoids and risk of ER+ PR - and ER - PR - breast cancers (16). In our study, weak inverse associations were observed for $\alpha$-carotene, $\beta$-carotene, and lutein/zeaxanthin and risk of ER - breast cancer. We found null associations between intakes of each of the 5 major carotenoids and risk of $\mathrm{ER}+, \mathrm{PR}+$, and $\mathrm{PR}-$ breast cancers. Previous studies examining breast cancer subtypes defined by ER 


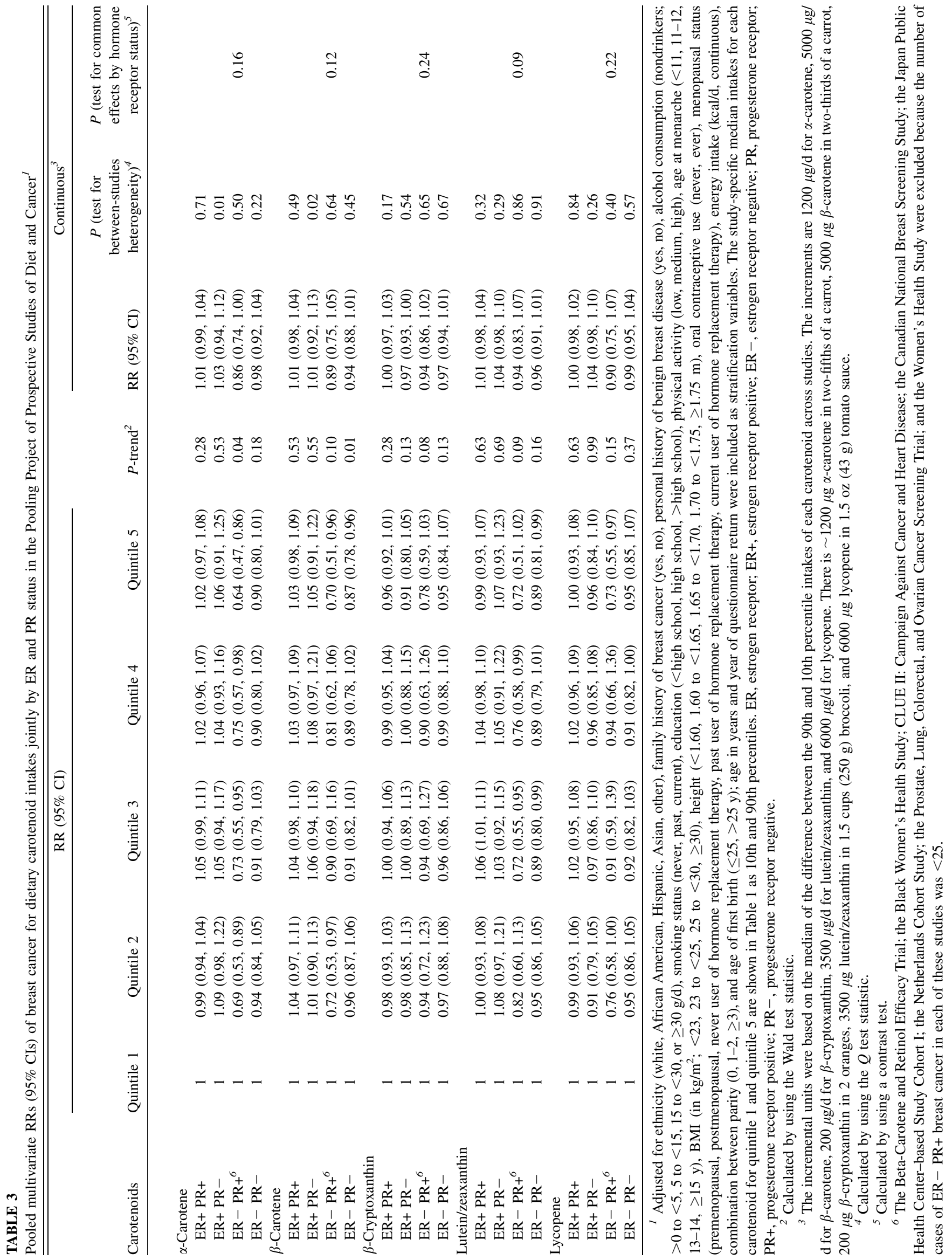


TABLE 4

Pooled multivariate RRs (95\% CIs) of breast cancer for dietary carotenoid intakes by ER status and menopausal status in the Pooling Project of Prospective Studies of Diet and Cancer ${ }^{1}$

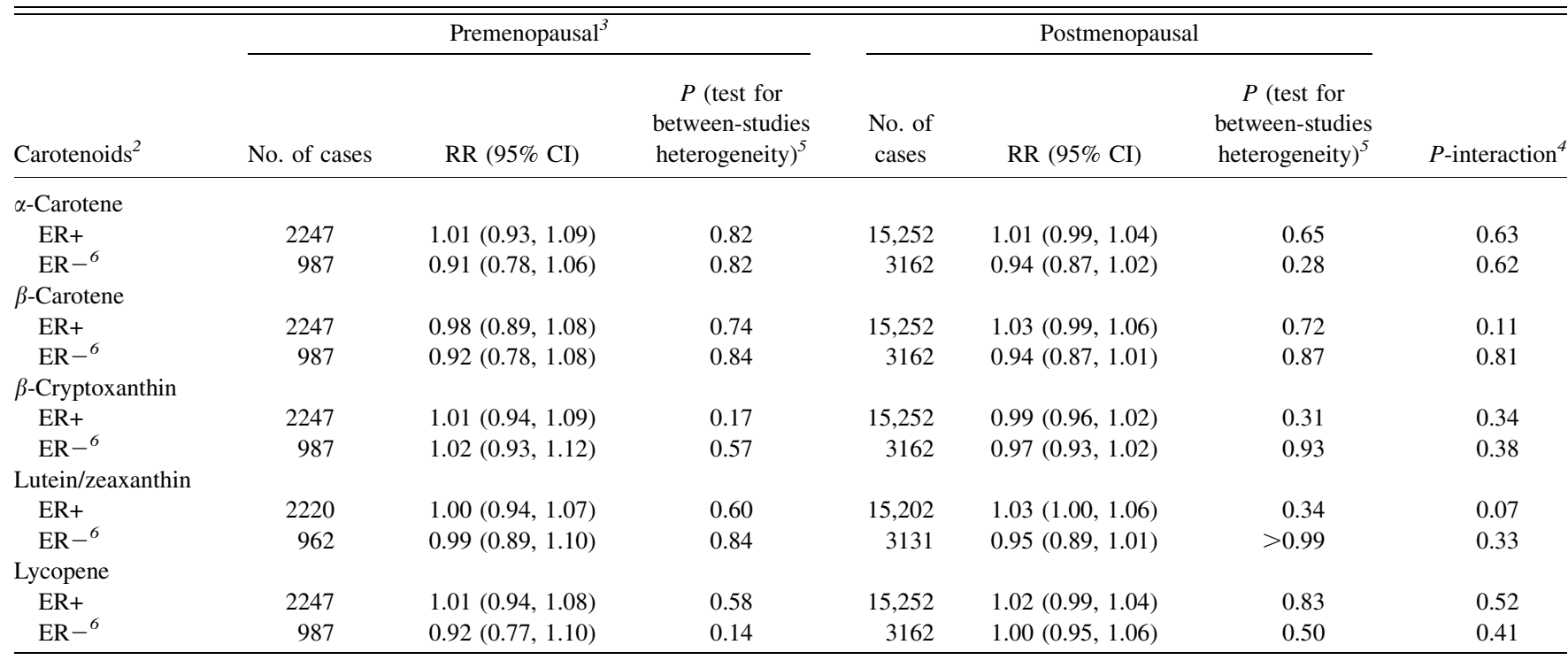

${ }^{1}$ RRs were adjusted for the covariates listed in Table 2. The Japan Public Health Center-based Study Cohort I was excluded from the analysis of lutein/ zeaxanthin because this variable was not measured in this study. ER, estrogen receptor; ER+, estrogen receptor positive; ER-, estrogen receptor negative.

${ }^{2}$ The incremental units were based on the median of the difference between the 90th and 10th percentile intakes of each carotenoid across studies. The increments are $1200 \mu \mathrm{g} / \mathrm{d}$ for $\alpha$-carotene, $5000 \mu \mathrm{g} / \mathrm{d}$ for $\beta$-carotene, $200 \mu \mathrm{g} / \mathrm{d}$ for $\beta$-cryptoxanthin, $3500 \mu \mathrm{g} / \mathrm{d}$ for lutein/zeaxanthin, and $6000 \mu \mathrm{g} / \mathrm{d}$ for lycopene. There is $\sim 1200 \mu \mathrm{g} \alpha$-carotene in two-fifths of a carrot, $5000 \mu \mathrm{g} \beta$-carotene in two-thirds of a carrot, $200 \mu \mathrm{g} \beta$-cryptoxanthin in 2 oranges, $3500 \mu \mathrm{g}$ lutein/ zeaxanthin in 1.5 cups $(250 \mathrm{~g})$ broccoli, and $6000 \mu \mathrm{g}$ lycopene in $1.5 \mathrm{oz}(43 \mathrm{~g})$ tomato sauce.

${ }^{3}$ The Iowa Women's Health Study, the Netherlands Cohort Study, and the Prostate, Lung, Colorectal, and Ovarian Cancer Screening Trial were excluded from the premenopausal analysis because all participants were postmenopausal. The Breast Cancer Detection Demonstration Project Follow-up Study, the Cancer Prevention Study II Nutrition Cohort, the NIH-AARP Diet and Health Study, and the Beta-Carotene and Retinol Efficacy Trial were excluded from the premenopausal analyses because of sparse stratum-specific case numbers.

${ }_{5}^{4}$ Calculated by using a Wald test.

${ }^{5}$ Calculated by using the $Q$ test statistic.

${ }^{6}$ The Nurses' Health Study II and the Women's Lifestyle and Health Study were excluded from the postmenopausal analyses because of sparse stratumspecific case numbers.

and PR status jointly have had limited power to examine ER $\mathrm{PR}+$ breast cancers because they have included $<40$ cases of ER - PR+ breast cancer $(16,17)$. Our study also showed null associations between intakes of each of the major carotenoids and risk of ER+ and PR+ and ER+ PR - breast cancers. However, intakes of $\alpha$-carotene, $\beta$-carotene, and lutein/zeaxanthin were associated with a slightly lower risk of $\mathrm{ER}-\mathrm{PR}-$ and ER - PR+ breast cancers.

Differences in clinical, pathological, and molecular features of breast cancer defined by ER and PR status suggest etiologic heterogeneity (9-13). The suggestive inverse associations observed for $\alpha$-carotene, $\beta$-carotene, and lutein/zeaxanthin intakes and risk of ER - breast cancer in our study support the possibilities that the effect of dietary factors, if any, might be confined to the less hormone-dependent ER - breast cancer and that carotenoid intakes might not have an important influence on ER+ breast cancer. However, experimental studies have shown that $\beta$-carotene and lycopene inhibit the growth of both ER+ and ER - cell lines (77).

Our pooled analysis had several strengths. In contrast with small studies with homogeneous populations, the large sample size and wide variation in carotenoid intakes allowed for more powerful analyses of the main effects of each of the major carotenoids with risk of breast cancer subtypes defined by ER/PR status and for evaluation of whether these associations were modified by several breast cancer risk factors. In addition, the prospective cohort design with high follow-up rate minimized the potential for recall or selection bias. We adjusted for multiple established or potential breast cancer risk factors to minimize confounding, and the age-adjusted results were almost identical to the multivariable adjusted results, which suggests that these associations were minimally confounded by these factors.

Our study had several limitations. Measurement error occurred as a result of assessing intake of the specific carotenoids with the use of FFQs. Most studies did not collect information on cooking methods for the specific fruit and vegetables consumed, and how fruit and vegetables are prepared and consumed may influence the bioavailability of carotenoids $(1,46)$. However, correlations of plasma concentrations of specific carotenoids with carotenoid intakes assessed by the FFQ and also by 2 wk of weighed diet records were very similar in the Nurses' Health Study and the Nurses' Health Study II (78). In addition to differences in chronic intakes, plasma concentrations of carotenoids are also influenced by differences in metabolism, including the recently described genetic variation in the $\beta$-carotene monoxygenase gene (79). Therefore, although bioavailability and differences in metabolism do not contribute to errors in measuring intake, they will affect plasma concentrations of carotenoids and probably the 


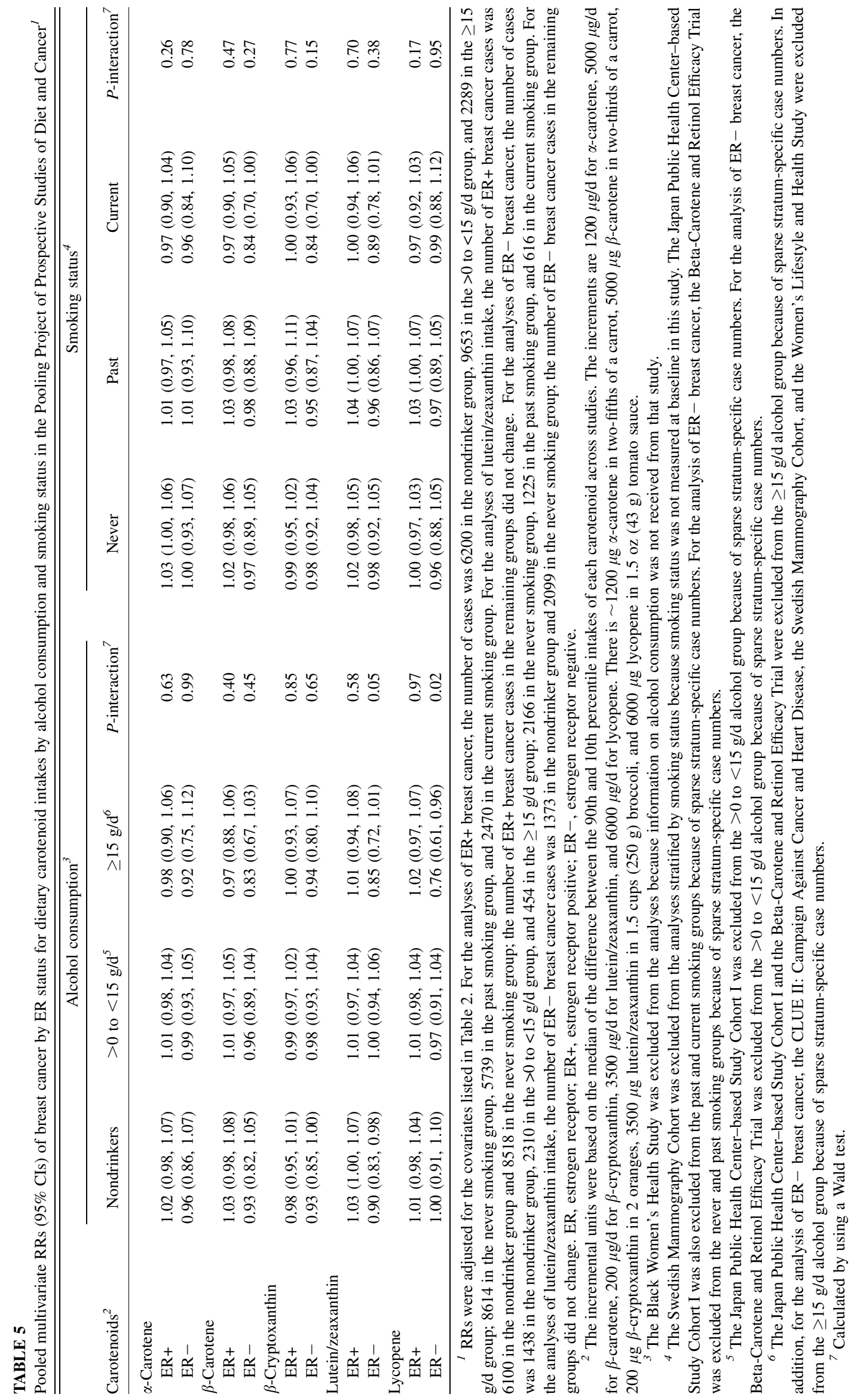


biological effects of intake. Year-to-year variation in diet likely exists. The use of a single questionnaire will therefore also contribute to error in the estimation of longer term intake, which is likely to be important in the etiology of breast cancer. In addition, correction for misclassification in intake estimates resulted in stronger inverse associations between intakes of $\alpha$-carotene, $\beta$-carotene, $\beta$-cryptoxanthin, and lutein/zeaxanthin and ERbreast cancer risk, which suggests that the true associations between dietary factors and disease risk might be underestimated substantially by using FFQs. In addition, because we measured carotenoid intake only at baseline, we cannot evaluate the possible effect of intakes during other life periods (eg, childhood) or changes in carotenoid intakes during follow-up.

An additional limitation of the study included the relatively high correlations between individual carotenoids, which made assessment of independent associations difficult. However, the observed results were essentially unchanged when we mutually adjusted intakes of the 5 carotenoids. Also, the observed associations with dietary carotenoids may have been due to their correlation with other bioactive constituents of fruit or vegetables. When we further adjusted for dietary folate and vitamin C intakes, the results were similar. However, the associations with specific carotenoids should be interpreted with caution because they may still be due to other unmeasured or unadjusted constituents of fruit and vegetables. Although an influence of the missing ER and PR status on the observed results is possible, no substantial differences were found between cases with and those without receptor status according to age, BMI, alcohol consumption, and some other reproductive factors. Our study populations are mainly of European origin, which limited our ability to examine the potential effect of carotenoid intakes on breast cancer risk in other ethnic groups, such as African American and Asian populations.

In summary, although intakes of specific carotenoids, except for $\beta$-cryptoxanthin, were not significantly associated with overall risk of breast cancer, $\alpha$-carotene, $\beta$-carotene, and lutein/ zeaxanthin intakes were associated with a modestly lower risk of $\mathrm{ER}-$, but not ER+, breast cancer. However, these results need to be interpreted with caution because it is unclear whether the observed associations are due to specific carotenoids or to other constituents of the same foods although when we adjusted for dietary vitamin $\mathrm{C}$ and folate intakes, other nutrients present in fruit and vegetables, the associations remained. Additional research is needed to identify the potential mechanisms that may account for the observed findings. Additional studies of plasma carotenoids and genetic variants in the carotenoid-metabolizing pathway in relation to ER - breast cancer may be useful in elucidating further the role of carotenoid intakes on the risk of ER - breast cancer.

We thank Sherry Yuan, Ruifeng Li, and Tao Hou for data management and statistical support. We are grateful for all participants in each study that was included in the Pooling Project.

The authors' responsibilities were as follows-XZ, SAS-W,WCW, and DS: designed research; XZ: analyzed the data and wrote the manuscript; DS, L Baglietto, L Bernstein, DAB, PAvdB, JEB, SMG, GGG, EG, GG, SEH, KJH, PLH-R, SY, MI, ML, PK, SCL, MLM, ABM, MLN, JRP, YP, KR, TER, JAR, LJS, JMS, ST, KV, EW, AW, WCW, SMZ, RGZ, and SAS-W: provided critical input in writing the manuscript; and SAS-W: had primary responsibility for final content. All authors read and approved the final manuscript. None of the authors declared a conflict of interest.

\section{REFERENCES}

1. Mangels AR, Holden JM, Beecher GR, Forman MR, Lanza E. Carotenoid content of fruits and vegetables: an evaluation of analytic data. J Am Diet Assoc 1993;93:284-96.

2. Peto R, Doll R, Buckley JD, Sporn MB. Can dietary beta-carotene materially reduce human cancer rates? Nature 1981;290:201-8.

3. Steinmetz KA, Potter JD. Vegetables, fruit, and cancer. II. Mechanisms. Cancer Causes Control 1991;2:427-42.

4. Rao AV, Rao LG. Carotenoids and human health. Pharmacol Res 2007; 55:207-16.

5. Frei B. Reactive oxygen species and antioxidant vitamins: mechanisms of action. Am J Med 1994;97(3A):5S-13S; discussion 22S-8S.

6. Clarke N, Germain P, Altucci L, Gronemeyer H. Retinoids: potential in cancer prevention and therapy. Expert Rev Mol Med 2004;6:1-23.

7. Hirsch K, Atzmon A, Danilenko M, Levy J, Sharoni Y. Lycopene and other carotenoids inhibit estrogenic activity of 17beta-estradiol and genistein in cancer cells. Breast Cancer Res Treat 2007;104:221-30.

8. World Cancer Research Fund, American Institute for Cancer Research Expert Panel. Food, nutrition and the prevention of cancer: a global perspective. Washington, DC: American Institute for Cancer Research, 2007.

9. Cotterchio M, Kreiger N, Theis B, Sloan M, Bahl S. Hormonal factors and the risk of breast cancer according to estrogen- and progesterone-receptor subgroup. Cancer Epidemiol Biomarkers Prev 2003;12: 1053-60.

10. Althuis MD, Fergenbaum JH, Garcia-Closas M, Brinton LA, Madigan MP, Sherman ME. Etiology of hormone receptor-defined breast cancer: a systematic review of the literature. Cancer Epidemiol Biomarkers Prev 2004;13:1558-68.

11. Huang WY, Newman B, Millikan RC, Schell MJ, Hulka BS, Moorman PG. Hormone-related factors and risk of breast cancer in relation to estrogen receptor and progesterone receptor status. Am J Epidemiol 2000;151:703-14.

12. Potter JD, Cerhan JR, Sellers TA, McGovern PG, Drinkard C, Kushi LR, Folsom AR. Progesterone and estrogen receptors and mammary neoplasia in the Iowa Women's Health Study: how many kinds of breast cancer are there? Cancer Epidemiol Biomarkers Prev 1995;4: 319-26.

13. Ursin G, Bernstein L, Lord SJ, Karim R, Deapen D, Press MF, Daling JR, Norman SA, Liff JM, Marchbanks PA, et al. Reproductive factors and subtypes of breast cancer defined by hormone receptor and histology. Br J Cancer 2005;93:364-71.

14. Smith-Warner SA, Spiegelman D, Ritz J, Albanes D, Beeson WL, Bernstein L, Berrino F, van den Brandt PA, Buring JE, Cho E, et al. Methods for pooling results of epidemiologic studies: the Pooling Project of Prospective Studies of Diet and Cancer. Am J Epidemiol 2006;163:1053-64.

15. Cooper JA, Rohan TE, Cant EL, Horsfall DJ, Tilley WD. Risk factors for breast cancer by oestrogen receptor status: a population-based case-control study. Br J Cancer 1989;59:119-25.

16. Cui Y, Shikany JM, Liu S, Shagufta Y, Rohan TE. Selected antioxidants and risk of hormone receptor-defined invasive breast cancers among postmenopausal women in the Women's Health Initiative Observational Study. Am J Clin Nutr 2008;87(4):1009-18.

17. Gaudet MM, Britton JA, Kabat GC, Steck-Scott S, Eng SM, Teitelbaum SL, Terry MB, Neugut AI, Gammon MD. Fruits, vegetables, and micronutrients in relation to breast cancer modified by menopause and hormone receptor status. Cancer Epidemiol Biomarkers Prev 2004;13: 1485-94.

18. Verhoeven DT, Assen N, Goldbohm RA, Dorant E, van 't Veer P, Sturmans F, Hermus RJ, van den Brandt PA. Vitamins C and E, retinol, beta-carotene and dietary fibre in relation to breast cancer risk: a prospective cohort study. Br J Cancer 1997;75:149-55.

19. Calle EE, Rodriguez C, Jacobs EJ, Almon ML, Chao A, McCullough ML, Feigelson HS, Thun MJ. The American Cancer Society Cancer Prevention Study II Nutrition Cohort: rationale, study design, and baseline characteristics. Cancer 2002;94:500-11.

20. Horn-Ross PL, Hoggatt KJ, West DW, Krone MR, Stewart SL, Anton H, Bernstei CL, Deapen D, Peel D, Pinder R, et al. Recent diet and breast cancer risk: the California Teachers Study (USA). Cancer Causes Control 2002;13:407-15.

21. Terry P, Jain M, Miller AB, Howe GR, Rohan TE. Dietary carotenoids and risk of breast cancer. Am J Clin Nutr 2002;76:883-8. 
22. Cho E, Spiegelman D, Hunter DJ, Chen WY, Zhang SM, Colditz GA, Willett WC. Premenopausal intakes of vitamins A, C, and E, folate, and carotenoids, and risk of breast cancer. Cancer Epidemiol Biomarkers Prev 2003;12:713-20.

23. George SM, Park Y, Leitzmann MF, Freedman ND, Dowling EC, Reedy J, Schatzkin A, Hollenbeck A, Subar AF. Fruit and vegetable intake and risk of cancer: a prospective cohort study. Am J Clin Nutr 2009;89:347-53.

24. Omenn GS, Goodman G, Thornquist M, Grizzle J, Rosenstock L, Barnhart S, Balmes J, Cherniack MG, Cullen MR, Glass A, et al. The beta-carotene and retinol efficacy trial (CARET) for chemoprevention of lung cancer in high risk populations: smokers and asbestos-exposed workers. Cancer Res 1994;54(suppl):2038s-43s.

25. Velie EM, Schairer C, Flood A, He JP, Khattree R, Schatzkin A. Empirically derived dietary patterns and risk of postmenopausal breast cancer in a large prospective cohort study. Am J Clin Nutr 2005;82: 1308-19.

26. Palmer JR, Rao RS, Adams-Campbell LL, Rosenberg L. Height and breast cancer risk: results from the Black Women's Health Study (United States). Cancer Causes Control 2001;12:343-8.

27. Genkinger JM, Platz EA, Hoffman SC, Comstock GW, Helzlsouer KJ. Fruit, vegetable, and antioxidant intake and all-cause, cancer, and cardiovascular disease mortality in a community-dwelling population in Washington County, Maryland. Am J Epidemiol 2004;160:1223-33.

28. Kobayashi M, Tsubono Y, Sasazuki S, Sasaki S, Tsugane S. Vegetables, fruit and risk of gastric cancer in Japan: a 10-year follow-up of the JPHC Study Cohort I. Int J Cancer 2002;102:39-44.

29. Giles GG, English DR. The Melbourne Collaborative Cohort Study. IARC Sci Publ 2002;156:69-70.

30. Prorok PC, Andriole GL, Bresalier RS, Buys SS, Chia D, Crawford ED, Fogel R, Gelmann EP, Gilbert F, Hasson MA, et al. Design of the Prostate, Lung, Colorectal and Ovarian (PLCO) Cancer Screening Trial. Control Clin Trials 2000;21(suppl):273S-309S.

31. Rexrode KM, Lee IM, Cook NR, Hennekens CH, Buring JE. Baseline characteristics of participants in the Women's Health Study. J Womens Health Gend Based Med 2000;9:19-27.

32. Kumle M, Weiderpass E, Braaten T, Persson I, Adami HO, Lund E. Use of oral contraceptives and breast cancer risk: the Norwegian-Swedish Women's Lifestyle and Health Cohort Study. Cancer Epidemiol Biomarkers Prev 2002;11:1375-81.

33. Zhang S, Hunter DJ, Forman MR, Rosner BA, Speizer FE, Colditz GA, Manson JE, Hankinson SE, Willett WC. Dietary carotenoids and vitamins A, C, and E and risk of breast cancer. J Natl Cancer Inst 1999;91:547-56.

34. Larsson SC, Bergkvist L, Wolk A. Dietary carotenoids and risk of hormone receptor-defined breast cancer in a prospective cohort of Swedish women. Eur J Cancer 2010;46(6):1079-85.

35. Kushi LH, Fee RM, Sellers TA, Zheng W, Folsom AR. Intake of vitamins $\mathrm{A}, \mathrm{C}$, and $\mathrm{E}$ and postmenopausal breast cancer. The Iowa Women's Health Study. Am J Epidemiol 1996;144:165-74.

36. Pedersen GS, Hogervorst JG, Schouten LJ, Konings EJ, Goldbohm RA, van den Brandt PA. Dietary acrylamide intake and estrogen and progesterone receptor-defined postmenopausal breast cancer risk. Breast Cancer Res Treat 2010;122:199-210.

37. Neuhouser ML, Patterson RE, Thornquist MD, Omenn GS, King IB, Goodman GE. Fruits and vegetables are associated with lower lung cancer risk only in the placebo arm of the $\beta$-carotene and retinol efficacy trial (CARET). Cancer Epidemiol Biomarkers Prev 2003;12:350-8.

38. Otani T, Iwasaki M, Yamamoto S, Sobue T, Hanaoka T, Inoue M, Tsugane S. Alcohol consumption, smoking, and subsequent risk of colorectal cancer in middle-aged and elderly Japanese men and women: Japan Public Health Center-based prospective study. Cancer Epidemiol Biomarkers Prev 2003;12:1492-500.

39. Baglietto L, Giles GG, English DR, Karahalios A, Hopper JL, Severi G. Alcohol consumption and risk of glioblastoma; evidence from the Melbourne Collaborative Cohort Study. Int J Cancer 2011;128(8):1929-34.

40. Leitzmann MF, Park Y, Blair A, Ballard-Barbash R, Mouw T, Hollenbeck AR, Schatzkin A. Physical activity recommendations and decreased risk of mortality. Arch Intern Med 2007;167:2453-60.

41. Kirsh VA, Hayes RB, Mayne ST, Chatterjee N, Subar AF, Dixon LB, Albanes D, Andriole GL, Urban DA, Peters U. Supplemental and dietary vitamin $\mathrm{E}$, beta-carotene, and vitamin $\mathrm{C}$ intakes and prostate cancer risk. J Natl Cancer Inst 2006;98:245-54.

42. Hedelin M, Lof M, Andersson TM, Adlercreutz H, Weiderpass E. Dietary phytoestrogens and the risk of ovarian cancer in the women's lifestyle and health cohort study. Cancer Epidemiol Biomarkers Prev 2011;20(2):308-17.

43. Chen J, Ruczinski I, Jorgensen TJ, Yenokyan G, Yao Y, Alani R, Liegeois NJ, Hoffman SC, Hoffman-Bolton J, Strickland PT, et al. Nonmelanoma skin cancer and risk for subsequent malignancy. J Natl Cancer Inst 2008;100:1215-22.

44. Boggs DA, Palmer JR, Spiegelman D, Stampfer MJ, Adams-Campbell LL, Rosenberg L. Dietary patterns and 14-y weight gain in African American women. Am J Clin Nutr 2011;94:86-94.

45. Holden JM, Eldridge AL, Beecher GR, Buzzard MI, Bhagwat S, Davis CS, Douglass LW, Gebhardt S, Haytowitz D, Schakel S. Carotenoid content of U.S. foods: an update of the database. J Food Compost Anal 1999;12:169-96.

46. Chug-Ahuja JK, Holden JM, Forman MR, Mangels AR, Beecher GR, Lanza E. The development and application of a carotenoid database for fruits, vegetables, and selected multicomponent foods. J Am Diet Assoc 1993;93:318-23.

47. Willett W. Nutritional epidemiology. 2nd ed. New York, NY: Oxford University Press, 1998.

48. Michaud DS, Giovannucci EL, Ascherio A, Rimm EB, Forman MR, Sampson L, Willett WC. Associations of plasma carotenoid concentrations and dietary intake of specific carotenoids in samples of two prospective cohort studies using a new carotenoid database. Cancer Epidemiol Biomarkers Prev 1998;7:283-90.

49. Flagg EW, Coates RJ, Calle EE, Potischman N, Thun MJ. Validation of the American Cancer Society Cancer Prevention Study II Nutrition Survey Cohort Food Frequency Questionnaire. Epidemiology 2000;11: $462-8$.

50. Tsubono Y, Sasaki S, Kobayashi M, Akabane M, Tsugane S. Food composition and empirical weight methods in predicting nutrient intakes from food frequency questionnaire. Ann Epidemiol 2001;11(3): 213-8.

51. Hodge AM, Simpson JA, Fridman M, Rowley K, English DR, Giles GG, Su Q, O'Dea K. Evaluation of an FFQ for assessment of antioxidant intake using plasma biomarkers in an ethnically diverse population. Public Health Nutr 2009;12:2438-47.

52. Kumanyika SK, Mauger D, Mitchell DC, Phillips B, Smiciklas-Wright $\mathrm{H}$, Palmer JR. Relative validity of food frequency questionnaire nutrient estimates in the Black Women's Health Study. Ann Epidemiol 2003;13:111-8.

53. Munger RG, Folsom AR, Kushi LH, Kaye SA, Sellers TA. Dietary assessment of older Iowa women with a food frequency questionnaire: nutrient intake, reproducibility, and comparison with 24-hour dietary recall interviews. Am J Epidemiol 1992;136:192-200.

54. Jain MG, Harrison L, Howe GR, Miller AB. Evaluation of a selfadministered dietary questionnaire for use in a cohort study. Am J Clin Nutr 1982;36:931-5.

55. Prentice RL. A case-cohort design for epidemiologic cohort studies and disease prevention trials. Biometrika 1986;73:1-11.

56. Rothman K. Modern epidemiology. Boston, MA: Little, Brown and Company, 1986.

57. Cox DR. Regression models and life-tables. J R Stat Soc B 1972;34: $187-220$.

58. Joffe MM, Rosenbaum PR. Invited commentary: propensity scores. Am J Epidemiol 1999;150:327-33.

59. Cepeda MS. The use of propensity scores in pharmacoepidemiologic research. Pharmacoepidemiol Drug Saf 2000;9:103-4.

60. Iami K, Van Dyk DA. Causal inference with general treatment regimes: generalizing the propensity score. J Am Stat Assoc 2004;99: 854-66.

61. Laird NM, Ware JH. Random-effects models for longitudinal data. Biometrics 1982;38:963-74.

62. DerSimonian R, Laird N. Meta-analysis in clinical trials. Control Clin Trials 1986;7:177-88.

63. Cochran WG. The combination of estimates from different experiments. Biometrics 1954;10:101-29.

64. Durrleman S, Simon R. Flexible regression models with cubic splines. Stat Med 1989;8:551-61.

65. Govindarajulu US, Spiegelman D, Thurston SW, Ganguli B, Eisen EA Comparing smoothing techniques in Cox models for exposure-response relationships. Stat Med 2007;26:3735-52.

66. Smith-Warner SA, Spiegelman D, Yaun S-S, van den Brandt PA, Folsom AR, Goldbohm RA, Graham S, Holmberg L, Howe GR, Marshall JR, et al. 
Alcohol and breast cancer in women: a pooled analysis of cohort studies. JAMA 1998;279:535-40.

67. Stram DO. Meta-analysis of published data using a linear mixed-effects model. Biometrics 1996;52:536-44.

68. Anderson TW. Introduction to multivariate statistics. 2nd ed. New York, NY: John Wiley \& Sons, 1984.

69. Hamling J, Lee P, Weitkunat R, Ambühl M. Facilitating meta-analyses by deriving relative effect and precision estimates for alternative comparisons from a set of estimates presented by exposure level or disease category. Stat Med 2008;27:954-70.

70. Barron BA. Effects of misclassification on estimation of relative risk. Biometrics 1977;33:414-8.

71. Greenland S. Variance-estimation for epidemiologic effect estimates under misclassification. Stat Med 1988;7:745-57.

72. Küchenhoff H, Mwalili SM, Lesaffre E. A general method for dealing with misclassification in regression: the misclassification SIMEX. Biometrics 2006;62:85-96.

73. Jarrow RA, Lando D, Turnbull SM. A Markov model for the term structure of credit risk spreads. Rev Financ Stud 1997;10:481-523.

74. Zhang S, Tang G, Russell RM, Mayzel KA, Stampfer MJ, Willett WC, Hunter DJ. Measurement of retinoids and carotenoids in breast adipose tissue and a comparison of concentrations in breast cancer cases and control subjects. Am J Clin Nutr 1997;66:626-32.

75. Rohan TE. Invited commentary: serum carotenoids and breast cancer. Am J Epidemiol 2001;153:1148-50, discussion 51.

76. van 't Veer P, Strain JJ, Fernandez-Crehuet J, Martin BC, Thamm M, Kardinaal AF, Kohlmeier L, Huttunen JK, Martin-Moreno JM, Kok FJ. Tissue antioxidants and postmenopausal breast cancer: the European Community Multicentre Study on Antioxidants, Myocardial Infarction, and Cancer of the Breast (EURAMIC). Cancer Epidemiol Biomarkers Prev 1996;5:441-7.

77. Prakash P, Russell RM, Krinsky NI. In vitro inhibition of proliferation of estrogen-dependent and estrogen-independent human breast cancer cells treated with carotenoids or retinoids. J Nutr 2001;131:1574-80.

78. Ascherio A, Stampfer MJ, Colditz GA, Rimm EB, Litin L, Willett WC Correlations of vitamin A and $\mathrm{E}$ intakes with the plasma concentrations of carotenoids and tocopherols among American men and women. J Nutr 1992;122:1792-801.

79. Leung WC, Hessel S, Meplan C, Flint J, Oberhauser V, Tourniaire F, Hesketh JE, von Lintig J, Lietz G. Two common single nucleotide polymorphisms in the gene encoding beta-carotene $15,15^{\prime}$-monoxygenase alter beta-carotene metabolism in female volunteers. FASEB J 2009;23: 1041-53. 